\title{
Melena - a rare clinical presentation in a patient with ruptured tubal ectopic pregnancy
}

\author{
Malkeet Kaur, Kavita Babbar, Devendra Singh, Aman Sharma \\ Corresponding author: Dr. Malkeet Kaur, Department of Obstetrics \& Gynecology, Apollo \\ hospitals, Bilaspur, Chhattisgarh, India; Email: drmalkeetkaur3@gmail.com
}

Distributed under Attribution-Non Commercial - Share Alike 4.0 International (CC BY-NC-SA 4.0)

\begin{abstract}
Ectopic pregnancy is a medical emergency which requires immediate intervention and may lead to death if left untreated. Patients usually present with abdominal pain, vaginal bleeding but may also present with signs of shock due to blood loss. We report a case of 32 years old patient who presented with melena as her chief complaint, did not mention history of amenorrhea but was later diagnosed as a case of ruptured ectopic tubal pregnancy.
\end{abstract}

Keywords: Ectopic rupture, fallopian tube pregnancy, melena.

Ectopic pregnancy occurs when the embryo attaches outside the uterine cavity. It is a potentially life threatening condition if timely intervention is not done. Usually the patient presents with abdominal pain and vaginal bleeding ${ }^{1}$. The patient may also show signs of shock with tachycardia, hypotension if there is an ongoing bleeding from the ectopic site if it has ruptured. Risk factors associated with ectopic pregnancy includes various pelvic inflammatory conditions, history of infertility, use of tobacco especially smoking, prior tubal procedures, chlamydia infection and interventions for infertility treatment. The most common site for attachment of embryo outside the uterus is in fallopian tubes referred to as tubal pregnancies ${ }^{2}$, however the attachment may occur in ovaries, cervix or it may be intrabdominal. History of missed periods along with ultrasound and serum beta human chorionic gonadotropin (HCG) helps confirming the diagnosis of ectopic pregnancy.

Melena refers to black tarry stools caused due to bleeding from upper gastrointestinal tract, with ulcers being the most common cause. Cases of melena due to ruptured ectopic pregnancy ${ }^{3}$ are rare and not reported frequently.

Case

A 32 years old married woman with average built presented to emergency department (ED) with complaints of black tarry stool, abdominal discomfort and distension for 3 days. She was being treated elsewhere but referred to our centre for gastroenterological consultation as her symptoms were persistent. Upon further evaluation she gave history of infertility for which she took treatment but eventually left the medications as she could not conceive despite treatment. Upon clinical evaluation, she was found to be having hypotension with her blood pressure readings to be 90 $\mathrm{mmHg}$ systolic and $60 \mathrm{mmHg}$ diastolic, her heart rate was around 120 per minute, respiratory rate around 24 per minute showing an oxygen saturation of $98 \%$ on room air. She was looking severely pale, minimally dehydrated and had an episode of melena while she was getting hospitalised in the ED. Upon systemic evaluation her chest was clear with no added sounds though she was having shortness of breath on mild exertion, heart sounds clearly audible without any murmurs or gallop rhythm, her abdomen had generalised mild tenderness with palpable fluid thrill, neurologically patient was alert with no signs of any focal deficit. She also stated that her urine output was adequate. She did not have any abnormal vaginal bleeding, her last menstrual cycle was for 3 days with normal flow and had occurred 25 days ago from her day of hospitalisation, she stated history of irregular menstrual cycle and had been on medications for infertility

Received: $10^{\text {th }}$ May 2020, Peer review completed: $17^{\text {th }}$ August 2020, Accepted: $1^{\text {st }}$ October 2020.

Kaur M, Babbar K, Singh D, Sharma A. Melena - a rare clinical presentation in a patient with ruptured tubal ectopic pregnancy. The New Indian Journal of OBGYN. 2021; 8(1): 146-48. 
treatment in the past.

She was started on intravenous (IV) crystalloids, IV proton pump inhibitor (PPI), blood products were arranged and her routine investigations were sent (compiled in table 1). In view of abdominal distension, an ultrasound was done which revealed a space occupying lesion in her right adnexa with gross ascites. This led to the suspicion of ruptured ectopic pregnancy and serum beta HCG was sent, an urgent urine pregnancy test was done which came to be positive.

\begin{tabular}{|l|}
\hline Table 1: Routine investigation report \\
\hline Complete blood count \\
Haemoglobin $-4.2 \mathrm{gm} \%$ \\
Haematocrit $-11.7 \%$ \\
Total leucocyte count $-7100 /$ cumm $(\mathrm{N}-84, \mathrm{~L}-12)$ \\
Platelets -2.00 lakhs $/$ cumm \\
\hline Renal function test \\
Urea - $24 \mathrm{mg} / \mathrm{dl}$ \\
Creatinine $-0.8 \mathrm{mg} / \mathrm{dl}$ \\
\hline Serum electrolytes \\
Sodium $-137 \mathrm{mmol} / \mathrm{L}$ \\
Potassium - $3.5 \mathrm{mmol} / \mathrm{L}$ \\
\hline Serum beta HCG $-12899 \mathrm{mIu} / \mathrm{ml}$ \\
\hline Urine test \\
Urine pregnancy test - positive \\
\hline
\end{tabular}

She was given packed red blood cells and rushed to operation theatre (OT), where a diagnostic laparoscopy was performed which revealed ruptured right tubal pregnancy (figure 1) with bleeding (figure 2). Further right salpingectomy was done, haemostasis was achieved and around four litres of hemoperitoneum was drained, peritoneal drain was placed and patient was shifted to critical care unit for further observation and management.

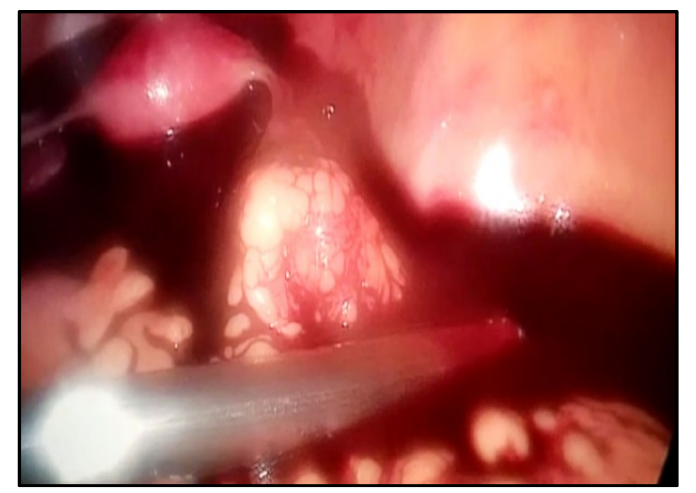

Figure 1: Right ruptured ectopic pregnancy with active bleeding The patient recovered well, there was no fresh episode of melena thus an upper gastrointestinal endoscopy was not done. She was shifted to ward the next day and discharged from hospital after 4 days with stable vitals.

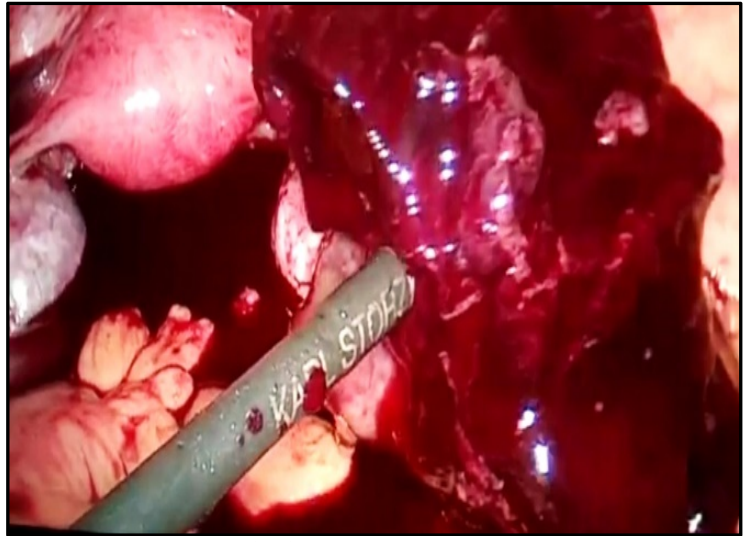

\section{Discussion}

Lower intestinal hemorrhage caused by ischemic necrosis of the small intestine induced by the compression of a fallopian tube hematoma in an ectopic pregnancy ${ }^{3}$ and severe rectal bleeding due to an ectopic pregnancy ${ }^{4}$ have been reported in the past. The exact pathophysiology of melena is not clear in patients with ruptured ectopic pregnancy and there have been very less cases reported with this presentation. Female patients in their fertile age groups can mimic various symptoms of other pathologies thus patients presenting from these age groups should be screened for a pregnancy.

This patient presented to our facility on the 3rd day after appearance of symptoms, prior to that she was treated at a local facility where she received supportive care along with antacid, she was referred to our facility for further management under a gastroenterologist. In our emergency department, he was evaluated and the preliminary impression was that this patient would be having an upper gastrointestinal (GI) bleed causing melena, leading to blood loss with signs of class III haemorrhage ${ }^{5}$. Further history and clinical evaluation were also in favour of GI bleeding as the patient did not have delayed periods. She had trouble conceiving for over past 10 years and had taken treatment for infertility which was unfruitful. Thus, the probability of she having a pregnancy was low, but the signs of free fluid in abdomen along with severe paleness and signs of haemorrhagic shock lead to the suspicion of a ruptured ectopic. This patient was resuscitated with IV fluids, blood products and immediately taken diagnostic laparoscopy with right salpingectomy, thus haemostasis was achieved and this patient was saved. 


\section{Conclusion}

Melena is a very rare presentation associated with ectopic pregnancy; this can be easily missed in the emergency department. The last menstrual periods should be always documented while obtaining patients history. These patients require immediate intervention as a ruptured ectopic pregnancy is a life threatening condition which can cause death within hours if left unattended.

Conflict of interest: None. Disclaimer: Nil.

\section{References}

1. Crochet JR, Bastian LA, Chireau MV. Does this woman have an ectopic pregnancy? the rational clinical examination systematic review. 2013. JAMA. 309 (16): 1722-9.

2. Cecchino GN, Araujo Júnior E, Elito Júnior J. Methotrexate for ectopic pregnancy: when and how. Archives of Gynecology and Obstetrics. 2014; 290 (3): 417-23.
3. Iwagaki H, Kitamura $\mathrm{M}$, Fuchimoto $\mathrm{S}$, Hamaya K, Kataoka K, Orita K. Frank melena in a patient with fallopian tube pregnancy. The Japanese Journal of Surgery. 1991; 21: 357- 9 .

4. Patterson SP, Frankum CE, Sexton H. Ectopic pregnancy causing massive rectal bleeding. South Med J. $1978 ; 71(11): 1449-50$.

5. Runciman WB, Skowronski GA. Pathophysiology of haemorrhagic shock. Anaesth Intensive Care. 1984; 12:193-205.

Malkeet Kaur ${ }^{1}$, Kavita Babbar ${ }^{2}$, Devendra Singh ${ }^{3}$, Aman Sharma ${ }^{4}$

${ }^{1}$ Senior RMO - Gynecology \& Obstetrics, Apollo hospitals, Bilaspur; ${ }^{2}$ Senior Consultant - Gynecology \& Obstetrics, Apollo hospitals, Bilaspur; ${ }^{3}$ Senior Consultant, Medical Gastroenterology, Apollo hospitals, Bilaspur; ${ }^{4}$ Senior Registrar - Emergency Medicine, Apollo hospitals, Bilaspur. 